\title{
Inclusion of isospin breaking effects in lattice simulations
}

\section{Antonin Portelli*}

School of Physics \& Astronomy, University of Southampton, SO17 1BJ, UK

E-mail: a.portelliesoton.ac.uk

Isospin symmetry is explicitly broken in the Standard Model by the mass and electric charge of the up and down quarks. These effects represent a perturbation of hadronic amplitudes at the percent level. Although these contributions are small, they play a crucial role in hadronic and nuclear physics. Moreover, as lattice computations are becoming increasingly precise, it is becoming more and more important to include these effects in numerical simulations. We summarize here how to properly define QCD and QED on a finite and discrete space-time so that isospin corrections to hadronic observables can be computed ab-initio and we review the main results on the isospin corrections to the hadron spectrum. We mainly focus on the recent work going beyond the electro-quenched approximation.

The 32nd International Symposium on Lattice Field Theory,

23-28 June, 2014

Columbia University New York, NY

* Speaker. 


\section{Motivation}

In an isospin symmetric world, the up $(u)$ and down $(d)$ quarks are identical particles. It is known ( $c f$. Table 1) than in Nature isospin symmetry is explicitly broken by the non-zero mass and electric charge differences of the $u$ and $d$ quarks. However, the effects of this breaking are expected to be small relative to typical strong interaction energies such as hadron masses. Indeed, it is clear that the light quark mass mass difference $\delta m=m_{u}-m_{d}$ represents one percent or less of any typical QCD energy scale. Similarly, the strength of the electromagnetic (EM) interaction relatively to the strong one is essentially given at low energy by the fine structure constant $\alpha \simeq 0.007$. For those reasons we can reasonably state that, for observables with a non-vanishing isospin symmetric part, isospin symmetry is a good approximation of reality with an $\mathrm{O}(1 \%)$ relative error.

Nevertheless, it is interesting to note that these small isospin breaking corrections are crucial to describe the structure of atomic matter in the Universe. Indeed, one particular effect of isospin symmetry breaking is the mass splitting between the proton $(p)$ and the neutron $(n)$. This mass difference is known experimentally with an impressive accuracy [1]:

$$
\Delta M_{N}=M_{n}-M_{p}=1.2933322(4) \mathrm{MeV}
$$

The sign of this splitting makes the proton and the hydrogen atom stable physical states. Also, the size of $\Delta M_{N}$ determine the phase space volume for the neutron $\beta$-decay $n \rightarrow p+e^{-}+\bar{v}_{e}$. At early times of the Universe ( $t \sim 1 \mathrm{~s}$ and $T \sim 1 \mathrm{MeV})$ and under standard assumptions ${ }^{1}$, the existence of $\beta$-decay allows to infer that the ratio of the number of neutrons and protons is approximatively equal to:

$$
\frac{n_{n}}{n_{p}} \simeq \exp \left(\frac{\Delta M_{N}}{T}\right)
$$

This ratio is one important initial conditions of Big Bang Nucleosynthesis. Also, in our actual Universe, $\beta$-decay and its inverse process are known to be responsible for the generation of a large majority of the stable nuclides chart though nuclear transmutation. Even if the nucleon isospin mass splitting is a well known quantity, predicting it from first principles is a difficult problem because of the complex non-perturbative interactions of quarks inside the nucleon. The proton carries an additional EM self-energy compared to the neutron, so just from QED one would expect to have $\Delta M_{N}<0$. However, the fact that the experimental value of $\Delta M_{N}$ has the opposite sign suggests that the strong isospin breaking effects are competing against the EM effects with a larger magnitude. This would mean that an important part of the structure of nuclear matter as we know it relies on

\begin{tabular}{|l|c|c|}
\cline { 2 - 3 } \multicolumn{1}{c|}{} & $u$ & $d$ \\
\hline Mass (MeV) [1] & $2.3\left({ }_{-0.5}^{+0.7}\right)$ & $4.8\left(_{-0.3}^{+0.7}\right)$ \\
Charge & $\frac{2}{3} e$ & $-\frac{1}{3} e$ \\
\hline
\end{tabular}

Table 1: Physical properties of the up and down quarks.

\footnotetext{
${ }^{1}$ The neutrino number density $n_{v} / n_{\gamma}$ is assumed to have the order of the baryon density number which is very small. This assumption is not valid anymore in some new physics scenarios but even in these hypothetical cases $n_{n} / n_{p}$ depends strongly on $\Delta M_{N}$.
} 
a subtle cancellation between the small EM and strong breaking effects of isospin symmetry in the nucleon system. Therefore, it is fundamental to have a theoretical understanding of the nucleon isospin mass splitting.

Considering that isospin breaking effects in the hadron mass spectrum are generally measured quite precisely, it is also interesting to understand how one can use this information to deduce the masses of the individual $u$ and $d$ quark masses. For example, it is important to know if $m_{u}=0$ could be a realistic solution to the strong CP problem. While recently ( $c f$. the FLAG review [2]) considerable progress has been made in determining precisely the average up-down quark mass $m_{u d}$ from first principles, such a computation is still missing for the individual masses. Because the kaon $(K)$ is a pseudo-Goldstone boson of chiral symmetry breaking, the isospin mass splitting $\Delta M_{K}^{2}=M_{K^{+}}^{2}-M_{K^{0}}^{2}$ is very sensitive to $\delta m$. But in order to extract $\delta m$, one has to understand how to subtract the EM contribution to this splitting. One well known result in this direction is Dashen's theorem [3] which states that, in the SU(3) chiral limit, the EM Kaon splitting is equal to the EM pion $(\pi)$ splitting:

$$
\Delta_{\mathrm{QED}} M_{K}^{2}=\Delta_{\mathrm{QED}} M_{\pi}^{2}+\mathrm{O}\left(\alpha m_{s}\right)
$$

This result is important because it is known [2] that with good accuracy, $\Delta_{\mathrm{QED}} M_{\pi}^{2} \simeq \Delta M_{\pi}^{2}$. The remaining question is: how large are the $\mathrm{O}\left(\alpha m_{s}\right)$ corrections in (1.3)? One way to quantify these corrections is to consider the dimensionless quantity $\varepsilon$ defined in [2] as follows:

$$
\varepsilon=\frac{\Delta_{\mathrm{QED}} M_{K}^{2}-\Delta_{\mathrm{QED}} M_{\pi}^{2}}{\Delta M_{\pi}^{2}}
$$

This quantity is constructed such that it vanishes in the $\mathrm{SU}(3)$ chiral limit. There were several attempts in the 1990s to compute these corrections analytically from effective theories which leaded to controversial results. It makes this quantity a good target for a lattice calculation.

The problems presented in this section, and more generally in any computation of isospin corrections to low-energy QCD observables, are difficult to solve because of the highly nonperturbative behavior of the strong interaction in this regime. It has been shown [2] that it is now possible to predict fundamental isospin symmetric QCD observables through lattice QCD simulations with a full control over the method's uncertainties. It is then reasonable to think that lattice simulations could be a reliable way to understand and compute isospin breaking effects. Moreover, besides the physical interest of these effects, actual lattice calculations are reaching a sub-percent precision on several standard observables and the assumption of isospin symmetry is becoming the dominant source of systematic uncertainty.

\section{Lattice QCD+QED}

In this section we review how to add EM interactions to lattice simulations. As we will see, the main difficulties comes from the singular infrared structure of QED. We explain a possible way to define QED in a finite volume and what are the associated finite-size effects. We then discuss the discretization of the theory and the simulation techniques used so far. 


\subsection{QCD+QED in a finite volume}

Let us consider a diagrammatic contribution $\mathscr{D}$ to a correlation function featuring a photon loop (e.g. the 1-loop part of the electron EM self-energy). In infinite volume, $\mathscr{D}$ will have the following form:

$$
\mathscr{D}_{\infty}=\int \frac{\mathrm{d}^{4} k}{(2 \pi)^{4}} \frac{1}{k^{2}} f\left(k, p_{1}, \ldots, p_{n}\right)
$$

The integral (2.1) may be ultraviolet (UV) divergent, which can be dealt with through renormalization. The photon pole at $k^{2}=0$ can also generate infrared (IR) divergences. However, (2.1) is not mathematically undefined per se, the undefined $k^{2}=0$ value of the integrand is just a point (set of measure 0) and can be ignored. Moreover, it is known that in some cases (e.g. the on-shell self-energy of a particle), this singularity is integrable. In a finite volume with temporal extent $T$ and spatial extent $L$, momenta become quantized in a way depending on the choice of boundary conditions. If one chooses periodic boundary conditions for the photon field, then the contribution (2.1) becomes:

$$
\mathscr{D}(T, L)=\frac{1}{T L^{3}} \sum_{k \in \mathrm{BZ}(T, L)} \frac{1}{k^{2}} f\left(k, p_{1}, \ldots, p_{n}\right)
$$

where:

$$
\mathrm{BZ}(T, L)=\frac{2 \pi}{T} \mathbb{Z} \times \frac{2 \pi}{L} \mathbb{Z}^{3}
$$

Now the expression (2.2) has an isolated, undefined contribution coming from the photon pole which cannot be summed in any way. As mentioned in [4, 5], this singularity is classically related to the fact that Gauss' law does not authorize a net charge to exist in a finite, periodic volume.

\subsubsection{Photon zero-mode subtraction schemes}

If one wants to keep periodic boundary conditions on the photon field, one possible solution to deal with the zero-mode singularity is to remove a subset of mode containing 0 from the finitevolume degrees of freedom. This will of course alter the physics in finite volume, but if the chosen subset converges in the infinite volume limit to a set of measure 0 then naively the physics in infinite volume remains unchanged. This is only a necessary condition, in principle one needs to check that the subtraction procedure does not accidentally couple the IR and UV structure of the theory which could introduce a complicated volume-dependent renormalization of the theory.

Naively, the most minimal zero-mode subtraction procedure is to set the $k=0$ mode of the photon field to 0. Following [6], we name the resulting theory $\mathrm{QED}_{\mathrm{TL}}$. This finite-volume prescription has been used for numerical calculations in $[7,8,9]$. Although this scheme is simple, it introduces some strong finite-volume effects which can be hard to control. Indeed, considering the $T \rightarrow+\infty$ limit at fixed $L$ of the $\mathrm{QED}_{\mathrm{TL}}$ version of (2.2), one obtains:

$$
\mathscr{D}_{\mathrm{QED}_{\mathrm{TL}}}(T, L)=\frac{1}{T L^{3}} \sum_{\substack{k \in \mathrm{BZ}(T, L) \\ k \neq 0}} f\left(k, p_{1}, \ldots, p_{n}\right) \underset{T \rightarrow+\infty}{\longrightarrow} \frac{1}{L^{3}} \int \frac{\mathrm{d} k_{0}}{2 \pi} \sum_{\mathbf{k} \in \mathrm{BZ}(L)} f\left(k, p_{1}, \ldots, p_{n}\right)
$$

where $\mathrm{BZ}(L)=\frac{2 \pi}{L} \mathbb{Z}^{3}$. Because it is one-dimensional, the integral in (2.4) might be IR divergent even in cases where its four-dimensional version converges. As an example, finite-volume effects 
on the 1-loop mass correction in spinor $\mathrm{QED}_{\mathrm{TL}}$ were computed in [6]:

$$
\begin{aligned}
m_{\mathrm{QED}_{\mathrm{TL}}}(T, L) \underset{T, L \rightarrow+\infty}{=} m\left\{1-q^{2} \alpha\left[\frac{\kappa}{2 m L}\left(1+\frac{2}{m L}\left[1-\frac{\pi}{2 \kappa} \frac{T}{L}\right]\right)\right.\right. \\
\left.\left.-\frac{3 \pi}{(m L)^{3}}\left[1-\frac{\operatorname{coth}(m T)}{2}\right]-\frac{3 \pi}{2(m L)^{4}} \frac{L}{T}\right]\right\}
\end{aligned}
$$

where $m$ is the infinite volume mass, $q$ is the charge in units of $e$ and $\kappa=2.83729 \ldots$ is a known numerical constant. This expansion is exact up to corrections that decay exponentially in the infinite volume limit. In this example we explicitly see a term proportional to $\frac{T}{L^{3}}$ which represents the IR divergence related to the limit (2.4). In conclusion, it appears that $\mathrm{QED}_{\mathrm{TL}}$ has two cumbersome properties. Firstly, the infinite-volume limit has to be taken with special care (i.e. by keeping $\frac{T}{L^{3}}$ bounded) and secondly this extrapolation depends on the aspect ratio $\frac{T}{L}$. As discussed in [6], the singularity of $\mathrm{QED}_{\mathrm{TL}}$ in the large $T$ limit can be explained in the following way. The photon zero-mode removal can be implement by adding a non-local term in the Lagrangian of the theory. This term couples values of the electromagnetic potential on different time-slices, breaking the reflection positivity of the action. So strictly speaking $\mathrm{QED}_{\mathrm{TL}}$ does not admit a quantum mechanical description and the divergence in $T$ is a symptom of the lack of a thermodynamic limit. This singular behavior has been discovered independently by the MILC collaboration [10].

An alternative to $\mathrm{QED}_{\mathrm{TL}}$ is to remove all spatial zero-modes, i.e. to set to zero all the photon modes $k$ with $\mathbf{k}=0$. This scheme is inspired from [11] where QED is formulated in a finite spatial volume directly with an infinite temporal dimension. We denote this prescription $\mathrm{QED}_{\mathrm{L}}[6]$. Because it does not couple field values on different time-slices, $\mathrm{QED}_{\mathrm{L}}$ has positive reflexivity and therefore a correct particle physics interpretation. In this theory, the finite-volume effects on the 1-loop mass correction of a spin $\frac{1}{2}$ particle are given by [6]:

$$
m_{\mathrm{QED}_{\mathrm{L}}}(T, L) \underset{T, L \rightarrow+\infty}{=} m\left\{1-q^{2} \alpha\left[\frac{\kappa}{2 m L}\left(1+\frac{2}{m L}\right)-\frac{3 \pi}{(m L)^{3}}\right]\right\}
$$

Compared to (2.5), this relation is now completely independent from the aspect ratio $\frac{T}{L}$. $\mathrm{QED}_{\mathrm{L}}$ has been used in electro-quenched simulations in [12] and in full simulations in [6].

The mass corrections in $\mathrm{QED}_{\mathrm{L}}$ and $\mathrm{QED}_{\mathrm{TL}}$ were compared to numerical quenched $\mathrm{QED}$ (which is exact at the 1-loop) simulations in [6] and perfect agreement is found between the simulations, (2.5) and (2.6). These results are summarized in Figure 1.

\subsubsection{Finite-volume effects on hadron masses}

All the previous statements were made for elementary particles which interact only through QED. Here we review how to generalize this discussion for hadrons, i.e. composite bound states of the strong interaction. All of the work presented here are computations performed in $\mathrm{QED}_{\mathrm{L}}$ with an infinite time dimension.

To study finite-volume effects on hadrons masses, one possible approach is to use low-energy effective theories of the strong interaction coupled to QED. This was done first for meson masses in the context of SU(3) partially-quenched chiral perturbation theory in [11]. The results of that work were studied numerically and generalized to an SU(2) plus heavy kaons theory in [12]. EM finitevolume corrections to meson, baryon, nuclei masses and to the hadronic vacuum polarization were 


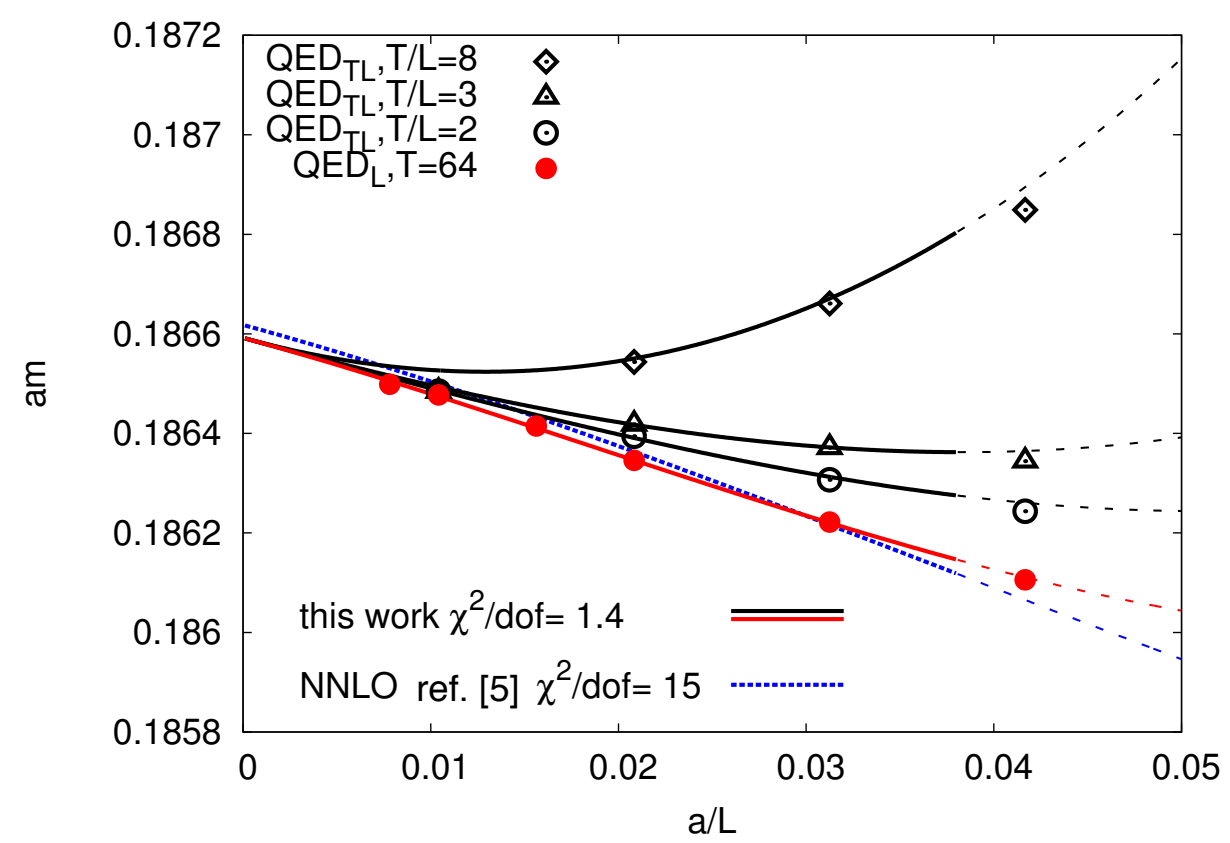

Figure 1: Finite-volume corrections to an elementary fermion mass in QED. The black (resp. red) points represent $\mathrm{QED}_{\mathrm{TL}}$ (resp. $\mathrm{QED}_{\mathrm{L}}$ ) quenched QED simulations (which is exact at $\mathrm{O}(\alpha)$ ). The black (resp. red) curves represent the theoretical prediction (2.5) (resp. (2.6)). The only fit parameter is the infinite-volume mass. The dashed blue line is the prediction from [5], the disagreement between this formula and the data is commented in section 2.1.2.

studied in [5] using non-relativistic effective theories. In this context, the finite-volume corrections appear as the elementary particle ones plus terms depending on the structure of the particle (radius, polarizabilities, ...).

However, there is a disagreement between the point-like limit of [5] and the QED prediction (2.6) concerning the fermion mass correction. The difference is a relative factor of 2 in the $\mathrm{O}\left[\alpha /(m L)^{3}\right]$ correction. The QED simulations presented in Figure 1 strongly favor (2.6). An explanation for this discrepancy has been recently proposed in [13]. In the non-relativistic limit, the particle and antiparticle degrees of freedom decouple and therefore in non-relativistic theories one does not expect the antiparticle modes to contribute to the particle mass corrections. However, as pointed out in [13], these modes contribute through the subtraction of the photon zero-modes in $\mathrm{QED}_{\mathrm{L}}$. Once properly added, this residual fermion-antifermion interaction generates a $\mathrm{O}\left[\alpha /(m L)^{3}\right]$ finite-volume correction which solves exactly the discrepancy with (2.6).

The structure of finite-volume corrections to hadron masses was also studied beyond the effective theory level [6]. In that work it is confirmed that the two first orders of the finite-volume expansion in $\frac{1}{m L}$ are universal and identical to the pure QED case. These terms are determined by gauge invariance, through the constraints on the electromagnetic form factors provided by the WardTakahashi identities, and follow from the analyticity properties of 1-particle irreducible Green's functions in the relevant quantum field theories. The structure contributions only enter at least at order $\mathrm{O}\left[\alpha /(m L)^{3}\right]$ which can be seen explicitly for the effective theories presented in $[11,12,5]$. The universality of the two first orders is an important information as it allows to impose these 
corrections analytically in lattice data analyses without introducing any model dependence.

\subsection{Lattice QED}

There are essentially two approaches to discretize QED: a naive, non-compact action where the gauge potential $A_{\mu}$ is still the field variable or a compact Wilson action similar to lattice QCD. By naive discretization we mean that the lattice action is defined as follows:

$$
S\left[A_{\mu}\right]=\frac{a^{4}}{4} \sum_{x, \mu, \nu}\left[\partial_{\mu} A_{v}(x)-\partial_{v} A_{\mu}(x)\right]^{2}
$$

where $a$ is the lattice spacing and $\partial_{\mu}$ is some first-order finite-difference operator. So far, except the starting project of the MILC collaboration [14], only the non-compact action has been used in the context of lattice QCD+QED simulations. One of the main motivations in making this choice comes from the fact that the non-compact action is free and still gauge-invariant ${ }^{2}$. On the other hand, the compact QED action introduces photon-photon interactions which are a pure discretization effect. However, there is in principle no conceptual problem in using compact QED. In both cases (although it is mandatory for the non-compact action) gauge fixing needs to be considered. Indeed, with electromagnetic interactions one might be interested in gauge variant amplitudes involving charged particles. In the non-compact case, gauge fixing is straightforward for Coulomb and Feynman gauges [6]. Moreover, as explained in [6], the non-compact action offers an interesting opportunity for Fourier acceleration of the hybrid Monte-Carlo (HMC) algorithm. The argument goes as follows: because the pure gauge theory is free, one can find a distribution for the HMC momenta which exactly cancels any autocorrelation in the Markov chain. Of course this is not correct anymore once quarks and gluons are coupled to the system. However, because of the weak coupling of QED it has been observed that using this particular momentum distribution still considerably reduces the autocorrelations coming from EM interactions.

\subsection{QCD+QED simulations}

Up to now, essentially two approaches have been used to perform lattice QCD+QED simulations. On the one hand, one can use the so-called electro-quenched approximation which consists in neglecting the EM contribution to the fermionic determinant (i.e. the sea quarks are electrically neutral). This approach is more cost effective but it is not possible to control reliably the quenching effects. On the other hand, one can consider the full theory. In the past couples of year several groups worked or started working on such simulations. We summarize below the effort done in both approaches.

\subsubsection{Electro-quenched approximation}

From the Monte-Carlo simulation point of view, the coupling of quenched QED (qQED) to QCD is fairly straightforward. For a given lattice QCD gauge configuration, one generates a pure gauge QED configuration (which is simply Gaussian distributed for the non-compact lattice action). Then the QED field is used to phase the QCD gauge links in the lattice Dirac operator inverted to obtain the valence quark propagators. Another approach has been used in [8]: in that

\footnotetext{
${ }^{2}$ this is not the case for non-compact lattice QCD [15]
} 
work the leading QED corrections are expressed as pure QCD expectation values in a perturbation theory fashion. In that framework, the electro-quenched approximation consist in neglecting the disconnected quark diagrams where the EM currents are self-contracted.

It is easy to show that the missing contributions to the fermionic determinant are suppressed by both the number of colors and SU(3) flavor symmetry. Using this fact and naive dimensional analysis, a quantity computed in the electro-quenched approximation is expected to suffer from a $\mathrm{O}(10 \%)$ quenching effect relatively to its electromagnetic corrections [9]. Also, partially quenched chiral perturbation theory allows to provide estimations consistent with the dimensional one for the light meson mass splittings [16, 17].

A summary of the different lattice QCD+qQED simulation projects can be found in [18]. Apart from a slight update from the MILC group [10], the actual situation is essentially identical concerning electro-quenched simulations.

\subsubsection{Full QCD+QED simulations}

There are essentially three possible ways to compute full QCD+QED correlation functions. Firstly, it is possible to compute directly the ratio of the QCD+QED to QCD fermionic determinant for each QCD configuration. These ratios can be then used to re-weight electro-quenched data. This technique was first proposed in [19] and applied in exploratory calculations [20, 21]. We see one major limitation of re-weighting techniques applied to QCD+QED: as the volume gets larger, the computational cost of the weights increases rapidly and the signal over noise ratio decreases. As discussed in section 2.1, it is important to reach large physical volumes in order to control the large finite-volume effects generated by QED.

A second approach already mentioned in the previous section is to perform a perturbative expansion in $\alpha$ and express the QED correction as pure QCD observables. The determinant contribution then appears as disconnected quark diagrams. The computation of these diagrams was never attempted in the context of computing EM corrections to hadronic amplitudes. However, identical diagrams contribute in other problems and they appear to be extremely difficult to compute. One can for example look at the recent study by the Mainz group [22] of the disconnected contributions to the hadronic vacuum polarization. This approach has the advantage of isolating specific perturbative contributions which can be useful for the control and understanding of IR divergences in hadronic processes [23].

Finally, one can generate new field configurations including both QCD and QED actions in the HMC process. Both QCDSF [24, 25] and MILC [14] have started simulations and BMWc achieved the first complete simulation program [6]. The specificities of each of the projects mentioned in this section is summarized in Table 2.

\section{Isospin corrections to the hadron spectrum}

In this section we review the different results concerning the isospin breaking corrections to the hadron spectrum. We first discuss the ambiguity of the separation of strong and EM contributions. Then we present the results concerning Dashen's theorem and quark masses and finally the hadron mass splittings. 


\begin{tabular}{|r|c|c|c|c|}
\hline collaboration & RBC-UKQCD & PACS-CS & QCDSF-UKQCD & BMWc \\
references & {$[20]$} & {$[21]$} & {$[24,25]$} & {$[6]$} \\
\hline fermion action & domain wall & Wilson & Wilson & Wilson \\
$N_{f}$ & $2+1$ & $1+1+1$ & $1+1+1$ & $1+1+1+1$ \\
method & re-weighting & re-weighting & HMC & HMC \\
$\min \left(M_{\pi}\right)(\mathrm{MeV})$ & 420 & 135 & 250 & 195 \\
$a(\mathrm{fm})$ & 0.11 & 0.09 & 0.08 & $0.06-0.10$ \\
$N_{a}$ & 1 & 1 & 1 & 4 \\
$L(\mathrm{fm})$ & 1.8 & 2.9 & $1.9-2.6$ & $2.1-8.3$ \\
$N_{\text {vol. }}$ & 1 & 1 & 2 & 11 \\
\hline
\end{tabular}

Table 2: Summary of full lattice QCD+QED simulation programs. The MILC program [14] is too preliminary to know its specifications and was not included in this table. The first line is the fermion action used. The second line is the number of flavors used in the gauge configuration generation. The third line gives the simulation method used. The forth line indicates the minimum pion mass reached. The fifth line is the range of lattice spacing used and the sixth line indicates their number. Similarly, the seventh and eighth lines are respectively the range and the number of lattice spatial extents used.

\subsection{Separation of QCD and QED contributions}

In all present work, only the leading isospin corrections to hadron masses are considered. These corrections can be written as follows:

$$
\Delta M_{X}=\alpha A_{X}+\delta m B_{X}+\mathrm{O}\left(\alpha^{2}, \alpha \delta m, \delta m^{2}\right)
$$

where $\Delta M_{X}$ is a given isospin mass splitting and $\delta m=m_{u}-m_{d}$. Then it is tempting to simply define the leading-order QED and QCD parts of the splitting:

$$
\Delta_{\mathrm{QED}} M_{X}=\alpha A_{X} \quad \text { and } \quad \Delta_{\mathrm{QCD}} M_{X}=\delta m B_{X}
$$

However, on has to be careful because of the following ambiguity: $\alpha$ and $\delta m$ depend on each other through radiative corrections. Moreover, $m_{u}$ and $m_{d}$ individually depend on $\alpha$ with a different coefficient because of the difference of their electric charges. Therefore, this ambiguity cannot be directly absorbed in higher-order isospin corrections. To make properly this QCD/QED separation, one has to provide a prescription that defines the $\delta m=0$ point. The difference between two prescriptions will be $\mathrm{O}\left(m_{u d} \alpha, m_{u d} \delta m\right)$ up to higher-order isospin corrections. Thus, with physical quark masses where $\delta m \simeq m_{u d}$ this discrepancy can be considered as higher-order isospin corrections. So as soon as a result is produced at the physical value of $m_{u d}$ and $\delta m$, it is reasonable to consider that the separation (3.1) is effectively unambiguous up to higher-order $\mathrm{O}(1 \%)$ corrections.

Several prescriptions to define the $\delta m=0$ point have been proposed in previous works. The most conceptually straightforward scheme is to renormalize the light quark masses in a given scheme (e.g. $\overline{\mathrm{MS}}$ or RI-MOM) at a given scale and to consistently express every quantity as a function of these renormalized masses. This prescription was used in [26, 12, 8]. Because renormalized quark masses can be difficult to compute, it is also interesting to consider prescriptions 
based on hadron masses. In [9], $\delta m$ was replaced by the mass squared difference between the connected $\bar{u} u$ and $\bar{d} d$ mesons $\Delta M^{2}=M_{\bar{u} u}^{2}-M_{\bar{d} d}^{2}$. Is it possible to show in partially-quenched chiral perturbation theory [16] that for physical quark masses, $\Delta M^{2}$ is directly proportional to $\delta m$ up to the $\mathrm{O}(1 \%)$ higher-order corrections. In the later work [6] from the same collaboration, the prescription $\Delta_{\mathrm{QED}} M_{\Sigma}=0$ was used, i.e. the $\Sigma^{+}-\Sigma^{-}$mass splitting is assumed to be proportional to $\delta m$. If these particles would be point-like, one would have $\Delta_{\mathrm{QED}} M_{\Sigma}=0$ exactly. The authors of [6] found $\Delta_{\mathrm{QED}} M_{\Sigma}$ statistically consistent with 0 and no more than $0.2 \mathrm{MeV}$ with $\Delta M^{2}=0$.

\subsection{Dashen's theorem and light quark masses}

Dashen's theorem corrections and individual up and down quark masses have been computed reliably only in the electro-quenched approximation. All existing results on Dashen's theorem correction $\varepsilon$ defined in (1.4) are presented in Figure 2. Two interesting comments can be make regarding these results. Firstly, although lattice results are still dominated by systematic uncertainties and suffer from an uncontrolled electro-quenching error, they look consistently spread around a common value. This contrasts significantly with the 1990's phenomenological determinations of this quantity. Secondly, the lattice results seems to favor a rather large $\mathrm{O}(70 \%)$ violation of Dashen's theorem.

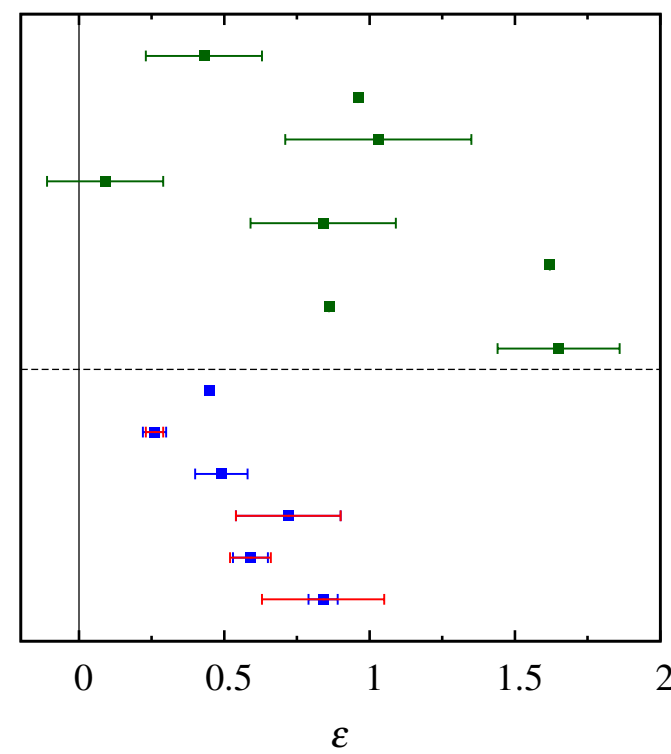

Maltman and Kotchan [27] (1990)

Donoghue et al. [28] (1993)

Bijnens [29] (1993)

Baur and Urech [30] (1996)

Bijnens and Prades [31] (1997)

Donoghue and Perez [32] (1997)

Gao et al. [33] (1997)

Moussallam [34] (1997)

Duncan et al. [7] (1996) (EQ, quenched)

RBC-UKQCD [26] (2007) (EQ)

RBC-UKQCD [12] (2010) (EQ)

RM123 [8] (2013) (EQ)

BMWc [35] (2014) (EQ, preliminary)

MILC [10] (EQ, preliminary)

Figure 2: Summary of the determination of Dashen's theorem violation $\varepsilon$ defined in (1.4). The green points represents analytical calculations from effective theory and the blue points results from lattice simulations. For the lattice results, the blue error bar is statistical and the red is systematic. "EQ" stands for electroquenched. Results are presented in chronological order.

Regarding the light quark mass ratio $m_{u} / m_{d}$, the lattice determinations of this number are summarized in Figure 3. Although there is a slight tension between the two most recent results [10,35], they both agree nicely with the values from the PDG [1] and FLAG [2] reviews. The only full QCD+QED result from PACS-CS [21] seems to deviate significantly from other determinations. This number is the result of an exploratory calculation using re-weighting techniques and has unknown systematic errors. We believe that this effect is more a systematic effect rather than an 
indication of a large see quark EM contribution. It is interesting to notice that all these results exclude strongly the $m_{u}=0$ solution to the strong CP problem.

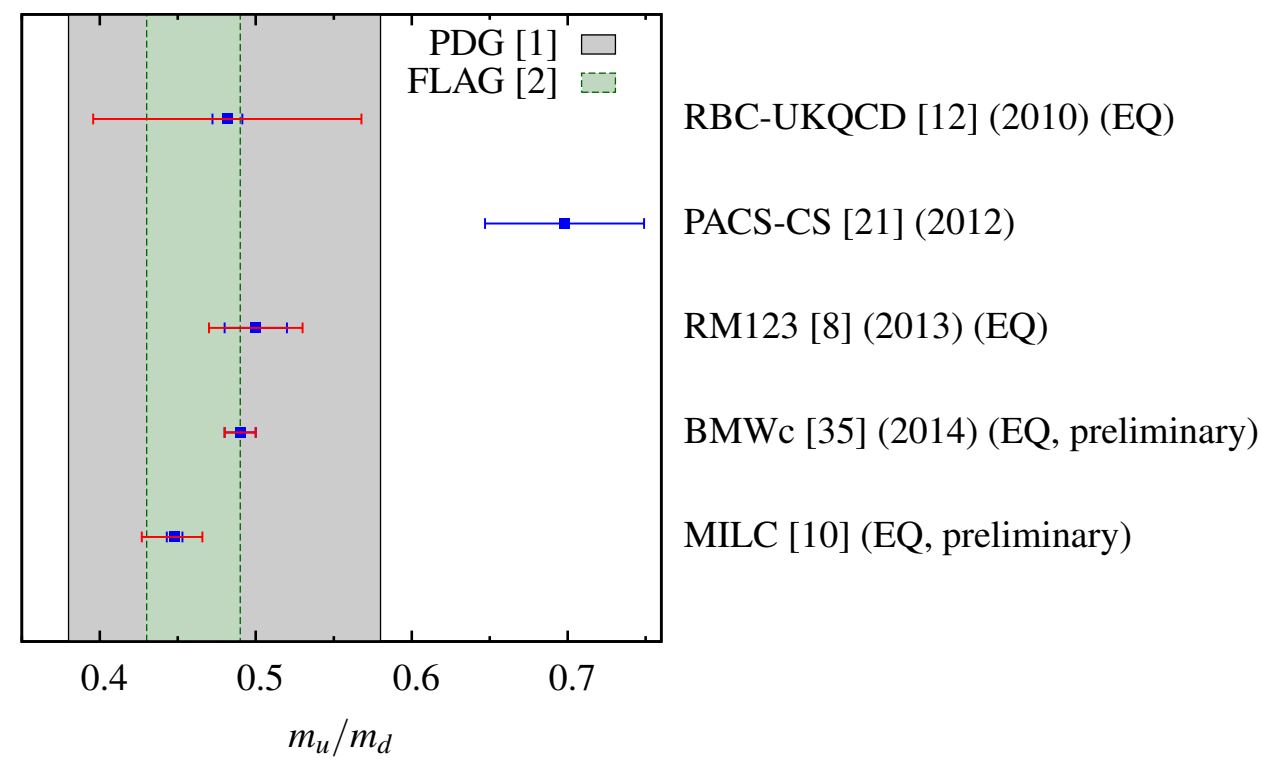

Figure 3: Summary of the calculations of $m_{u} / m_{d}$ light quark mass ratio. Plotting conventions are identical to the one used in Figure 2.

\subsection{Isospin mass splittings in the hadron spectrum}

The main novelty concerning the calculation of the isospin correction to the hadron spectrum is the high-precision determination of isospin mass splittings of the octet baryons, the $D$ meson and the newly discovered $\Xi_{c c}$, from the BMWc group [6]. These splittings where computed using full QCD+QED simulations including an active charm quark in the sea. A summary of these results can be found in Figure 4. The splittings obtained in this work are in very good agreement with experimental values. It is interesting to notice that the $\Xi$ baryon splitting is obtained with a precision higher than the experimental measurement. Moreover, the unknown $\Xi_{c c}$ splitting needed by charm spectrum experiments ${ }^{3}$ is predicted accurately.

The QCDSF-UKQCD collaboration also aim at studying isospin corrections to the octet baryon spectrum. This group have started generating full QCD+QED gauge ensembles [24, 25] to determine the corrections to the spectrum. The analysis is performed using the same technique based on SU(3) flavor symmetry as used in their previous pure QCD work [36]. The same collaboration also achieved the first lattice determination of the mixing between $\Sigma^{0}$ and $\Lambda^{0}$ baryons [37] which is authorized once isospin symmetry is broken. They obtained the mass splitting between the two particles with a precision of $\mathrm{O}(10 \%)$, in good agreement with the experimental value.

Finally we summarize the theoretical determination of the nucleon splitting, including the QCD and QED separation, in Figure 5. The most recent full QCD+QED results [6, 25] are in very good agreement and indicate that the crucial value of the nucleon mass splitting is indeed the result of a subtle cancellation between the QCD and QED contributions.

\footnotetext{
${ }^{3}$ cf. e.g. http://www.ectstar.eu/sites/www.ectstar.eu/files/talks/after-jurgen-feb13.pdf
} 


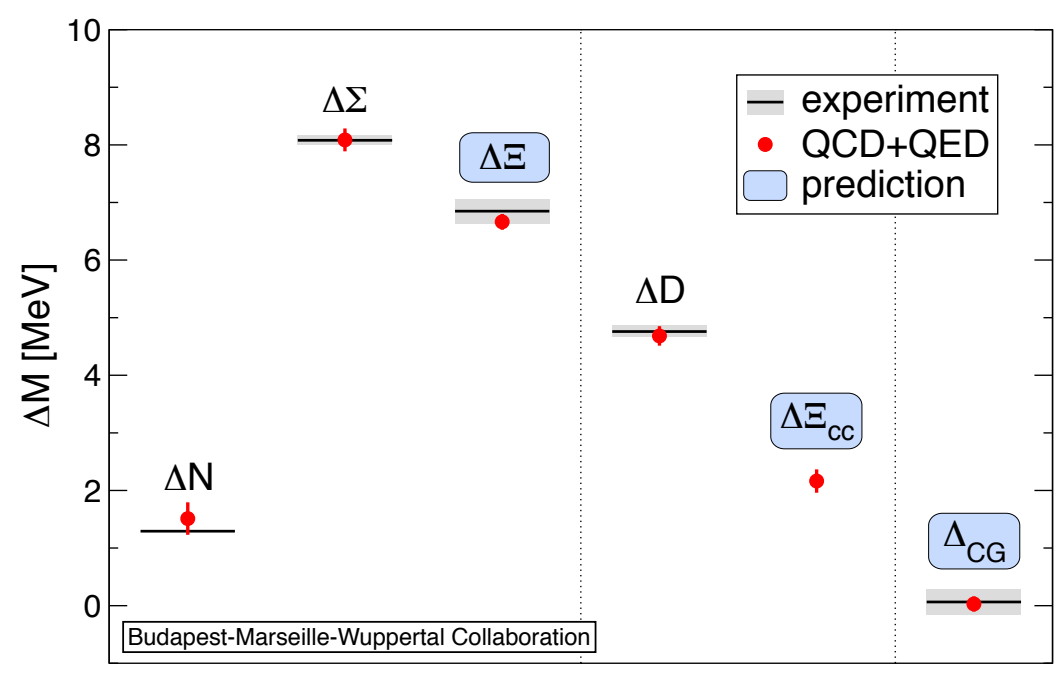

Figure 4: Summary of the results from [6]. $\Delta_{\mathrm{CG}}=\Delta M_{N}-\Delta M_{\Sigma}+\Delta M_{\Xi}$ is the correction to the ColemanGlashow relation.

\section{Conclusion and perspective}

Lattice QCD simulations in the isospin limit are reaching a precision of $\mathrm{O}(1 \%)$ and below on important observables and isospin breaking effects are becoming the dominant source of systematic uncertainty. Therefore, it is becoming crucial to introduce isospin breaking effects in lattice simulation in order to provide more stringent theoretical constraints on the Standard Model. The main challenge in this task is the inclusion of EM interactions.

The difficulty with QED comes from the IR singular structure of the theory. Defining correctly the theory in a box is non-trivial for several reasons. Firstly, momentum quantization can introduce hard singularities coming from the photon field zero-mode if one uses periodic boundary conditions. Subtracting the zero-mode of the field is a possible solution. However, fixing the zero-mode constitutes a non-local constraint on the theory which can break some important properties such as reflection positivity. It is shown in [6] that the zero-mode subtraction proposed by Hayakawa \& Uno [11] has a correct quantum mechanical interpretation. Beyond the zero-mode subtraction, QED in a finite volume suffers from large, power-like finite-size effects because of the long-range of the interaction. These effects are now well understood for hadron masses. The two first orders in the infinite-volume expansion are entirely determined by gauge invariance and can be computed analytically $[6,5]$. Several effective theory descriptions of the higher-order, structure dependent corrections have been worked out $[11,12,5,13]$.

In its recent work, the BMWc group [6] achieved the first complete lattice calculation featuring full QCD and QED interactions, an active sea charm quark and a full control over the different sources of uncertainty. The corrections to the baryon octet and charm spectrum are obtained precisely, in good agreement with experimental measurements. These simulations represent an important step toward fully non-perturbative, high precision, predictions of Standard Model observables. The corrections to the light meson spectrum, necessary to determine the individual up and down 


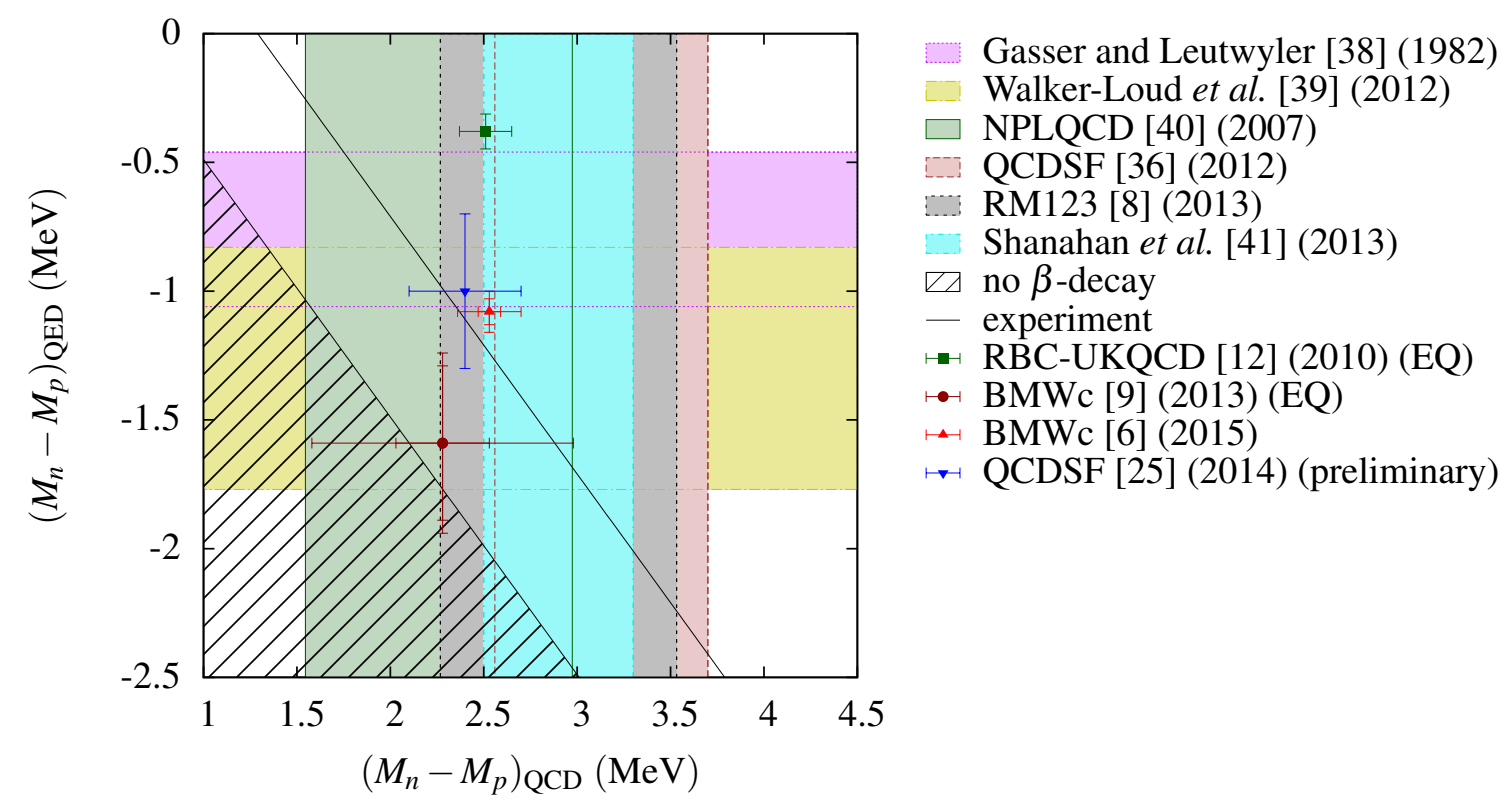

Figure 5: Review of the theoretical determinations of the nucleon mass splitting. Results represented by a band correspond to works where only the QCD or QED contribution has been determined. Where possible, error bars are represent statistical and systematic uncertainties. The "no $\beta$-decay" region is defined by $M_{n}-M_{p}<m_{e}$ where $m_{e}$ is the electron mass. "EQ" stands for electro-quenched.

light quark masses are still known only through the electro-quenched approximation.

It is now important to consider more complex quantities than hadron masses. Adding isospin breaking effects to the determination of hadronic decay widths is crucial to obtain high precision constraints on the flavor structure of the Standard Model (e.g. through sub-percent determinations of the CKM matrix coefficients). With EM interactions, matrix elements are significantly harder to determine than energy levels. Indeed, such quantity can feature IR divergences that are physically cancelled by the addition of real soft photons in the final state. Recently, a proposal has been made [23] to deal with such divergences in the case of meson decays. Also, QCD+QED simulations can be used to perform non-perturbative computation of the hadronic corrections to the muon anomalous magnetic moment [42]. This quantity features an interesting discrepancy between theory and experiment and it is important to reinforce the theoretical prediction to support the experimental effort.

\section{Acknowledgments}

I am supported by the UK STFC grants ST/J000396/1 and ST/L000296/1. I would like to thank the members of the Budapest-Marseille-Wuppertal collaboration for their precious help and support. I also thank C. Bernard, R. Horsley, G. Schierholz and P. Shanahan for kindly sharing their results with me at the time of the conference. I am grateful to L. Del Debbio, C. Lehner, T. Izubuchi, A. Patella, A. Ramos, C.T. Sachrajda and F. Sanfilippo for useful discussions. 


\section{References}

[1] K. Olive et al. (PDG). Review of Particle Physics. Chin. Phys. C 38 p. 090001 (2014). doi:10.1088/1674-1137/38/9/090001

[2] S. Aoki et al. (FLAG). Review of lattice results concerning low-energy particle physics. Eur. Phys. J. C 74(9) p. 2890 (2014). doi:10.1140/epjc/s10052-014-2890-7

[3] R. Dashen. Chiral $\mathrm{SU}(3) \otimes \mathrm{SU}(3)$ as a symmetry of the strong interactions. Phys. Rev. 183(5) pp. 1245-1260 (1969). doi:10.1103/PhysRev.183.1245

[4] A. Portelli et al. (BMWc). Electromagnetic corrections to light hadron masses. PoS(Lattice 2010)121 (2010). arXiv:1011.4189

[5] Z. Davoudi and M. J. Savage. Finite-volume electromagnetic corrections to the masses of mesons, baryons, and nuclei. Phys. Rev. D 90(5) p. 054503 (2014). doi:10.1103/PhysRevD.90.054503

[6] S. Borsanyi et al. (BMWc). Ab initio calculation of the neutron-proton mass difference. Science 347 (2015). doi: 10 .1126/science. 1257050

[7] A. Duncan, E. Eichten and H. Thacker. Electromagnetic splittings and light quark masses in lattice QCD. Phys. Rev. Lett. 76(21) pp. 3894-3897 (1996). doi:10.1103/PhysRevLett.76.3894

[8] G. M. de Divitiis et al. (RM123). Leading isospin breaking effects on the lattice. Phys. Rev. D 87(1) p. 114505 (2013). doi:10.1103/PhysRevD.87.114505

[9] S. Borsanyi et al. (BMWc). Isospin splittings in the light baryon octet from lattice QCD and QED. Phys. Rev. Lett. 111(25) p. 252001 (2013). doi:10.1103/PhysRevLett.111.252001

[10] S. Basak et al. (MILC). Finite-volume effects and the electromagnetic contributions to kaon and pion masses. PoS(Lattice 2014) (2014). arXiv:1409.7139

[11] M. Hayakawa and S. Uno. QED in finite volume and finite size scaling effect on electromagnetic properties of hadrons. Prog. Theor. Phys. 120(3) pp. 413-441 (2008). doi:10.1143/PTP.120.413

[12] T. Blum et al. (RBC-UKQCD). Electromagnetic mass splittings of the low lying hadrons and quark masses from 2+1 flavor lattice QCD+QED. Phys. Rev. D 82 p. 094508 (2010). doi:10.1103/PhysRevD.82.094508

[13] Z. Fodor et al. Quantum electrodynamics in finite volume and nonrelativistic effective field theories (2015). arXiv:1502.06921

[14] R. Zhou and S. Gottlieb (MILC). Dynamical QCD+QED simulation with staggered quarks. PoS(Lattice 2014) (2014). arXiv:1411.4115 
[15] E. Seiler, I. O. Stamatescu and D. Zwanziger. Monte Carlo simulation of noncompact QCD with stochastic gauge fixing. Nucl. Phys. B 239(1) pp. 177-200 (1984). doi:10.1016/0550-3213(84)90089-0

[16] J. Bijnens and N. Danielsson. Electromagnetic corrections in partially quenched chiral perturbation theory. Phys. Rev. D 75(1) p. 014505 (2007). doi:10.1103/PhysRevD.75.014505

[17] A. Portelli et al. (BMWc). Systematic errors in partially-quenched QCD plus QED lattice simulations. PoS(Lattice 2011)136 (2012). arXiv:1201.2787

[18] A. Portelli. Review on the inclusion of isospin breaking effects in lattice calculations. PoS(KAON13)023 (2013). arXiv:1307.6056

[19] A. Duncan, E. Eichten and R. Sedgewick. Computing Electromagnetic Effects in Fully Unquenched QCD 71(9) p. 094509 (2005). doi : 10.1103 /PhysRevD . 71.094509

[20] T. Ishikawa et al. Full QED+QCD low-energy constants through reweighting. Physical Review Letters 109(7) p. 072002 (2012). doi:10.1103/PhysRevLett. 109.072002

[21] S. Aoki et al. (PACS-CS). $1+1+1$ flavor QCD + QED simulation at the physical point 86(3) p. 034507 (2012). doi:10.1103/PhysRevD. 86.034507

[22] A. Francis et al. The leading disconnected contribution to the anomalous magnetic moment of the muon. Proceedings of the XXXII International Symposium on Lattice Field Theory, PoS(Lattice 2014) (2014). arXiv:1411. 7592

[23] N. Carrasco et al. QED Corrections to Hadronic Processes in Lattice QCD (2015). arXiv:1502.00257

[24] R. Horsley et al. (QCDSF-UKQCD). Electromagnetic splitting of quark and pseudoscalar meson masses from dynamical QCD + QED. PoS(LATTICE 2013)499 (2013). arXiv:1311.4554

[25] G. Schierholz (QCDSF-UKQCD). Private communication (2014)

[26] T. Blum et al. (RBC-UKQCD). Determination of light quark masses from the electromagnetic splitting of pseudoscalar meson masses computed with two flavors of domain wall fermions. Phys. Rev. D 76(11) p. 114508 (2007). doi:10.1103/PhysRevD . 76.114508

[27] K. Maltman and D. Kotchan. Chiral log corrections to pseudoscalar electromagnetic selfenergies and the reliability of Dashen's theorem. Mod. Phys. Lett. A 5(29) pp. 2457-2464 (1990). doi:10.1142/S0217732390002857

[28] J. F. Donoghue, B. R. Holstein and D. Wyler. Electromagnetic self-energies of pseudoscalar mesons and Dashen's theorem. Phys. Rev. D 47(5) pp. 2089-2097 (1993). doi:10.1103/PhysRevD.47.2089 
[29] J. Bijnens. Violations of Dashen's theorem. Phys. Lett. B 306(3-4) pp. 343-349 (1993). doi:10.1016/0370-2693(93)90089-z

[30] R. Baur and R. Urech. Corrections to Dashen's theorem. Phys. Rev. D 53(11) pp. 6552-6557 (1996). doi:10.1103/PhysRevD.53.6552

[31] J. Bijnens and J. Prades. Electromagnetic corrections for pions and kaons : masses and polarizabilities. Nucl. Phys. B 490(1-2) pp. 239-271 (1997). doi:10.1016/S0550-3213(97)00107-7

[32] J. F. Donoghue and A. F. Perez. The electromagnetic mass differences of pions and kaons. Phys. Rev. D 55(11) pp. 7075-7092 (1997). doi:10.1103/PhysRevD . 55 . 7075

[33] D.-N. Gao, B. A. Li and M.-L. Yan. Electromagnetic mass splittings of $\pi$, $a_{1}, \quad K, \quad K_{1}(1400)$, and $K^{*}(892)$. Phys. Rev. D 56(7) pp. $4115-4132$ (1997). doi:10.1103/PhysRevD.56.4115

[34] B. Moussallam. A Sum rule approach to the violation of Dashen's theorem. Nucl. Phys. B 504(1-2) pp. 381-414 (1997). doi:10.1016/S0550-3213 (97) 00464-1

[35] Budapest-Marseille-Wuppertal collaboration. Up and down quark masses and corrections to Dashen's theorem from lattice $Q C D$ and $Q E D$ (in preparation)

[36] R. Horsley et al. (QCDSF-UKQCD). Isospin breaking in octet baryon mass splittings. Phys. Rev. D 86(44) p. 114511 (2012). doi:10.1103/PhysRevD. 86.114511

[37] R. Horsley et al. A lattice determination of Sigma - Lambda mixing (2014). arXiv:1411.7665

[38] J. Gasser and H. Leutwyler. Quark masses. Phys. Rep. 87(3) pp. 77-169 (1982). doi:10.1016/0370-1573(82)90035-7

[39] A. Walker-Loud, C. E. Carlson and G. A. Miller. The electromagnetic self-energy contribution to $M_{p}-M_{n}$ and the isovector nucleon magnetic polarizability. Phys. Rev. Lett. 108(23) p. 232301 (2012). doi:10.1103/PhysRevLett.108.232301

[40] S. R. Beane, K. Orginos and M. J. Savage (NPLQCD). Strong-isospin violation in the neutronproton mass difference from fully-dynamical lattice $Q C D$ and PQQCD. Nucl. Phys. B 768(12) pp. 38-50 (2007). doi:10.1016/j.nuclphysb.2006.12.023

[41] P. E. Shanahan, A. W. Thomas and R. D. Young. Strong contribution to octet baryon mass splittings. Phys. Lett. B 718(3) pp. 1148-1153 (2013). doi:10.1016/j.physletb.2012.11.072

[42] T. Blum, S. Chowdhury, M. Hayakawa and T. Izubuchi. Hadronic Light-by-Light Scattering Contribution to the Muon Anomalous Magnetic Moment from Lattice QCD. Phys. Rev. Lett. 114(1) p. 012001 (2015). doi:10.1103/PhysRevLett.114.012001 